\title{
Étude exploratoire de l'utilisation des TICE en soutien aux pédagogies actives en contexte d'enseignement universitaire
}

Anastassis Kozanitis, Université du Québec à Montréal

kozanitis.anastassis@uqam.ca

Claude Quevillon, Université du Québec à Montréal quevillon_lacasse.claude@uqam.ca

Dans le cadre d'une étude exploratoire, un questionnaire en ligne a été envoyé aux professeur(e)s et chargé(e)s de cours universitaires de différentes universités québécoises afin de dégager un portrait de leur utilisation des TICE en support aux pédagogies actives proposées aux étudiants dans leurs cours. Les données descriptives recueillies chez les participants volontaires $(n=119)$ ont été analysées, puis complétées au moyen d'une analyse en composantes principales pour affiner les relations qui sont ressorties entre les variables retenues.

Mots-clés: Pédagogie universitaire, utilisation des TICE, modélisation des pratiques d'enseignement universitaire, analyse en composantes principales, profils d'enseignant

\section{Introduction}

Ces dernières années, les pratiques pédagogiques en classe en contexte universitaire ont été marquées par une évolution vers des pédagogies actives et l'utilisation croissante des technologies de l'information et de la communication pour l'enseignement (TICE) (Charbonneau, 2013; Tessier, 2012). Cette évolution est présente dans plusieurs régions du monde. Toutefois, l'étendue et la fréquence d'utilisation peuvent varier considérablement, non seulement par région, mais aussi par discipline (Guennoun et Benjelloun, 2016). Malgré tout, le traditionnel exposé magistral continue d'être la méthode d'enseignement la plus utilisée, quoiqu'elle tende à diminuer au profit des stratégies d'enseignement qui impliquent une plus grande participation de la part des étudiants (Kozanitis et Desbiens, 2016). À ce sujet, il est de plus en plus reconnu que l'apprentissage, la rétention des connaissances et la réussite augmentent lorsque les étudiants participent activement aux tâches et qu'ils sont placés dans des situations où ils sont encouragés à partager leurs conclusions et leurs réflexions (Freeman et al., 2014). Pour ce qui est de I'utilisation des TICE à des fins pédagogiques, la littérature scientifique montre qu'elle croît rapidement, tant chez les enseignants que chez les étudiants en contexte universitaire (Buckley, Pitt, Norton et Owens, 2010, Venkatesh et al., 2014).Toutefois, bien que les enseignants d'université utilisent 
régulièrement les TICE, la plupart d'entre eux se limitent à une utilisation restreinte de celles-ci, c'est-àdire à la transmission de l'information (Kirkup et Kirkwood, 2005). Dans le contexte de l'augmentation de leur utilisation, il vaut la peine de se demander si les TICE enrichissent, améliorent ou approfondissent l'apprentissage qui a lieu à l'université. Du point de vue des étudiants, ce qu'il faut vraiment considérer, ce n'est pas de savoir si les enseignants utilisent les TICE, mais plutôt comment ils les utilisent (Karsenti et Collin, 2011).

Quoi qu'il en soit, de nombreuses études révèlent un impact positif des TICE sur l'enseignement et l'apprentissage (Paivandi et Espinosa, 2013; Raby et al., 2011). Parmi les principaux avantages pour les étudiants universitaires, on constate un accès facile, gratuit et décentralisé à l'information (Higgins, Xiao et Katsipataki, 2012; Rogers, 2001), la possibilité de collaboration ou de coopération synchrone, asynchrone et délocalisée (Cruz, Cutchis et Honeyford, 2012), la prise en compte des préférences dans les manières d'apprendre (Battalio, 2009), ainsi que la réception d'une rétroaction immédiate (Johnson et Johnson, 2014). Cet accès favorise la responsabilité et l'engagement de l'étudiant envers son apprentissage et sa réussite (Brooks, 2016; Turney, Robinson, Lee et Soutar, 2009). De plus, les activités en ligne et les outils technologiques favorisent l'autorégulation et l'autonomie des étudiants (Lameul et Loisy, 2014). Elles ont également un impact positif sur l'intérêt des étudiants pour le cours et son contenu (Karsenti et al., 2011).Ainsi, de façon générale, les TICE favorisent l'apprentissage en profondeur chez les étudiants universitaires (Rogers, 2004) et mènent au développement de processus cognitifs de haut niveau (Monsakul, 2008).

Par ailleurs, il semble que l'utilisation combinée des pédagogies actives et des TICE participe à un apprentissage en profondeur grâce, notamment, à un accès régulier et cohérent aux ressources disponibles en ligne, car elles facilitent les interactions entre l'enseignant et les étudiants (Knight, 2010). Cependant, nous ne pouvons attribuer aux TICE la capacité d'améliorer l'apprentissage sans tenir compte de la manière dont elles sont utilisées et du contexte dans lequel elles sont utilisées (Céci, 2018; Romero et Laferrière, 2015). Plusieurs facteurs peuvent influencer la qualité de l'apprentissage. Nous devons donc considérer la situation dans son ensemble, en tenant compte des acteurs impliqués, des ressources disponibles et des activités d'apprentissage.

Maintenant, si les méthodes d'enseignement actives semblent susciter l'intérêt d'un nombre croissant de professeurs d'université, y compris ceux qui travaillent dans la formation professionnelle (Bachy, Lebrun et Smidts, 2010), on ne sait pas encore comment ces situations d'enseignement-apprentissage sont liées à l'utilisation des TICE. De même, la littérature souligne que les enseignants universitaires n'ont pas nécessairement reçu de formation pour l'utilisation des technologies numériques en enseignement (Collin et al., 2015). Qui plus est, ces formations ne favoriseraient pas la pratique réflexive à propos de l'utilisation des TICE (Roussel et al., 2017).Étant donné les avantages des TICE pour l'enseignement, et compte tenu de l'impact positif des pédagogies actives pour l'apprentissage en profondeur et pour encourager les processus cognitifs de haut niveau, surtout en contexte universitaire, il nous semble intéressant de savoir si les enseignants utilisent les TICE lorsqu'ils proposent des situations d'apprentissage actif et de quelle manière ils les utilisent en soutien à ces pédagogies actives. Pour ce faire, nous proposons d'explorer les questions suivantes: comment les enseignants universitaires exploitent-ils le potentiel des TICE pour augmenter et accroître l'efficacité des pédagogies actives? Quels sont les facteurs qui influencent l'utilisation des TICE par les enseignants universitaires? Les enseignants universitaires ont-ils reçu une formation sur les pédagogies actives ou sur les TICE? Quelles sont la nature et la durée des formations suivies? Quel est le profil d'utilisateur TICE des enseignants universitaires?

L'objectif général de la présente étude est de répertorier les TICE qui sont utilisées lors des situations d'enseignement en classe impliquant les pédagogies actives. Pour y parvenir, un questionnaire en ligne a été envoyé à des professeurs et chargés de cours de trois universités canadiennes (deux 
francophones et une anglophone) afin de mieux connaître leur utilisation des pédagogies actives dans leur enseignement, d'une part, et leur intégration des TICE en support à ces pédagogies actives, d'autre part. Nous avons également cueilli certaines données sociodémographiques, ce qui a permis d'analyser les facteurs potentiels qui pourraient influencer l'intégration des TICE en contexte universitaire. Pour alléger le texte, le terme enseignant désignera désormais à la fois les professeurs et les chargés de cours universitaires, à moins d'indication contraire. Les prochains paragraphes présentent le cadre conceptuel élaboré à partir de définitions existantes des concepts de pédagogies actives et de TICE en contexte universitaire. Puis, nous exposons brièvement la méthodologie avant de présenter les résultats et les analyses qui ont été réalisées. Finalement, une discussion nous permettra de revenir sur les résultats obtenus.

\section{Cadre conceptuel}

Comme nous nous intéressons à l'utilisation des TICE en soutien aux pédagogies actives en enseignement universitaire, il convient de définir ces deux concepts centraux. Des propositions sont également mises en avant pour distinguer et contraster la variété des pédagogies actives. Une classification en fonction des usages et de l'utilisation des TICE est également proposée.

\section{Définitions du concept de pédagogies actives}

La plupart des auteurs ayant proposé des définitions concernant les pédagogies actives conviennent qu'elles se caractérisent par une implication active de la part des étudiants. Dans ce contexte, l'essence du qualificatif « actif »renvoie à l'intention pédagogique de l'enseignant de susciter un engagement et une participation active des étudiants dans le processus d'apprentissage. Or, il ne peut s'agir de pédagogies actives en l'absence de son corollaire qu'est l'apprentissage actif. D'ailleurs, Bonwell et Eisen (1991) définissent les pédagogies actives à partir du pôle apprenant, soit comme toute stratégie qui demande aux étudiants de faire quelque chose et de réfléchir à ce qu'ils font. Selon ces auteurs, l'apprentissage actif présente les caractéristiques suivantes: l'activité des étudiants en classe ne se limite pas à l'écoute de l'enseignant; les activités sont variées (résolution de problèmes, débats, recherche, expérimentation, travail collaboratif, etc.); elles mettent davantage l'accent sur le développement des compétences que sur la transmission de l'information; et elles conduisent au développement de capacités cognitives d'ordre supérieur. Prince (2004) clarifie et simplifie la situation en déclarant que deux conditions sont nécessaires pour considérer qu'il y a un apprentissage actif en classe. La première, comme le disent Bonwell et Eisen (1991), exige que les étudiants accomplissent une activité autre que celle d'écouter le professeur et de prendre des notes. La seconde est que l'activité soit en lien avec les concepts, la matière ou les objectifs d'apprentissage du cours. De plus, les recherches de Carr, Palmer et Hagel (2015), qui ont mis au point un outil pour mesurer l'apprentissage actif, montrent que les interactions interpersonnelles entre les étudiants sont un élément fortement associé à l'apprentissage actif. Selon Smart et Csapo (2007), les méthodes d'enseignement qui mettent l'accent sur l'apprentissage actif offrent des possibilités d'interaction et d'engagement cognitif à partir d'activités contrôlées. En ce sens, Wanner (2015) a constaté que l'engagement cognitif et l'apprentissage actif sont étroitement liés, en plus d'être appréciés par les étudiants. Les deux sont de plus en plus considérés comme des préalables à un apprentissage significatif, pour stimuler l'utilisation de processus cognitifs supérieurs, comme la pensée critique, et pour promouvoir le développement de compétences professionnelles (Miller et al., 2011).

De leur côté, Freeman et al. (2014)ont dégagé leur définition des pédagogies actives à partir d'entrevues avec des acteurs scolaires, c'est-à-dire à partir des représentations des enseignants au sujet du concept 
d'apprentissage actif, soit le pôle apprenant des pédagogies actives. À l'instar de Petress (2008), comme le soulignent Carr, Palmer et Hagel (2015), la définition retenue par Freeman et al. (2014) de l'apprentissage actif s'appuie sur la démonstration de son contraire, soit l'apprentissage passif: « processus d'apprentissage qui engage les étudiants dans des activités ou des discussions en classe, par opposition à l'écoute passive d'un cours magistral, et mobilisant des processus cognitifs de haut niveau et (souvent) le travail de groupe. » (Freeman et al., 2014, p. 8413, traduction libre). Notons par ailleurs, à la suite de Carr, Palmer et Hagel (2015), que les activités relevant de pédagogies actives sont multiples, allant de la discussion en petit groupe à l'apprentissage expérientiel, en passant par la résolution de problèmes, les simulations et l'apprentissage par les pairs, pourvu que les conditions de base, énoncées dans la définition retenue, soient respectées.

\section{Diversité des pédagogies actives}

En effet, il existe une grande variété de stratégies d'enseignement ou de méthodes pédagogiques qui permettent de concevoir des situations d'apprentissage actif. Sans prétendre à l'exhaustivité, nous pouvons mentionner que l'apprentissage par problèmes et l'apprentissage par projet sont des exemples éloquents qui semblent de plus en plus utilisés dans les carrières scientifiques et techniques (Chenet al., 2008). Il est possible de regrouperles différentes pédagogies actives selon plusieurs caractéristiques ou critères. Par exemple, la figure 1 présente une adaptation du modèle de Prince (2004), qui propose de les situer sur un continuum allant des stratégies à dominante magistrale (magistro-centrée), situées à gauche, aux stratégies centrées davantage sur les étudiants (pédo-centrée), situées du côté droit. Deux critères sont utilisés pour décider de l'emplacement des stratégies sur le continuum. Le premier est d'identifier qui, del'enseignant ou des étudiants, fait la plus grande partie de l'activité en classe. Du côté du magistro-centré, la plupart des activités sont faites par l'enseignant. Du côté pédo-centré, ce sont les étudiants qui font la majeure partie de l'activité. Le second critère est la durée dans le temps de l'activité associée à la stratégie pédagogique. Par exemple, des exposés magistraux combinés avec des activités d'apprentissage actif à court terme, comme l'intégration d'une discussion de type «think-pair-share » qui durera moins de 10 minutes, seront situées du côté magistro-centré. Certaines stratégies pouvant durer entre une heure et trois heures, comme la méthode des cas, le puzzle ou le jeu de rôle, se situeront à mi-chemin sur le continuum. Enfin, les activités de plus longue durée, telles que l'apprentissage par projet, seront situées à l'extrême droite.

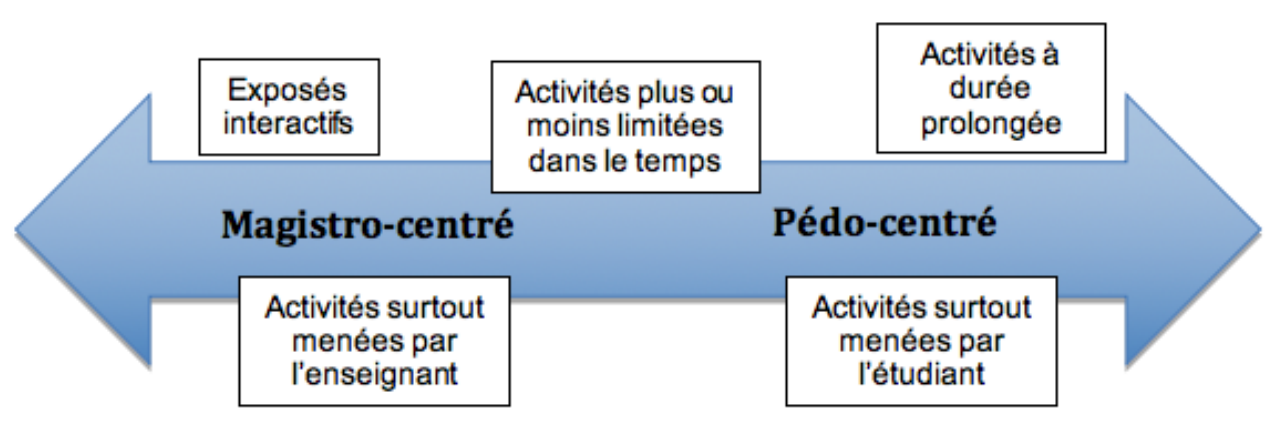

Figure 1. Continuum des stratégies d'apprentissage actif adapté de Prince (2004) 
Ce modèle permet de situer les stratégies d'enseignement en fonction de la durée de l'activité et de l'auteur principal de cette activité. Toutefois, il ne permet pas d'identifier la nature ou le type d'activité réalisé.ll nous semble dès lors nécessaire de trouver un moyen d'organiser les diverses stratégies d'enseignement qui prenne en considération, en plus de ces deux critères, un critère supplémentaire se référant à l'activité cognitive ou comportementale des étudiants. Ce critère souscrit pleinement à la définition retenue des pédagogies actives. Par contre, nous n'avons pas été en mesure de trouver de référence, scientifique ou autre, qui proposent une organisation des pédagogies actives qui tienne compte de ces trois critères. Cela dit, une analyse des documents repérés a permis de distinguer les pédagogies actives selon les trois critères retenus et de mener à un regroupement de celles-ci en trois groupes:1) l'apprentissage réflexif-interactif, 2) l'apprentissage inquisitif et 3) l'apprentissage expérientiel. Avant de décrire plus en détail ce regroupement, il convient de souligner que toute tentative de classification des pédagogies actives n'est pas sans poser certaines difficultés. La première a trait au choix des termes retenus pour l'identification des groupes. En effet, en voulant trouver une terminologie appropriée, nous avons plutôt constaté qu'il existe une pluralité et une multiplicité des termes employés par la communauté scientifique. De surcroît, l'utilisation des termes peut varier selon les auteurs, la région du monde ou encore le domaine d'application. La deuxième difficulté, en lien avec cette première, est l'absence de définition unique et univoque des concepts que sont censés représenter ces termes. À ce sujet, la principale lacune de la plupart des articles consultés est l'absence d'une référence phare pour l'identification et le discernement précis du concept en question. Pour ajouter à la confusion, certains auteurs peuvent employer, dans le même article, plus d'un terme quand ils réfèrent à un concept, comme s'il s'agissait de synonymes, sans pour autant en justifier la permutabilité. Une troisième difficulté émane du fait que la majorité des publications soit en langue anglaise, ce qui, au moment de la traduction des termes anglophones vers le français, peut occasionner un risque d'interprétation. À cela s'ajoute la difficulté de déterminer avec précision et sans erreur le groupe d'appartenance des pédagogies actives puisque les critères de discrimination ne sont pas suffisamment précis ou mutuellement exclusifs. Cela est particulièrement vrai pour les critères de la durée et de la nature de l'activité.

En dépit de ces difficultés, le regroupement proposé s'appuie sur une traduction à partir de la terminologie la plus fréquemment utilisée pour identifier les trois groupes. Ce regroupement n'a certainement pas la prétention d'être exhaustif, puisque d'autres critères pourraient s'ajouter et influencer les décisions. Les prochains paragraphes décrivent chaque groupe et présentent des exemples de stratégies d'enseignement qui leur sont associés.

Le premier groupe, que nous avons nommé apprentissage réflexif-interactif, recouvre des stratégies d'apprentissage actif situées du côté gauche du continuum de Prince. Ces stratégies sont généralement utilisées en salle de classe, en combinaison avec des méthodes d'enseignement plus traditionnelles, comme l'exposé magistral. II s'agit d'activités de courte durée (quelques minutes) que l'enseignant peut proposer afin de susciter chez l'étudiant une réflexion à propos d'un concept ou une idée en lien avec la leçon ou la matière du cours, l'intention étant de les amener à mobiliser des habiletés cognitives d'ordre supérieur (analyse, évaluation, discrimination, anticipation, déduction, pensée critique, etc.) de façon ponctuelle. Elles peuvent être réalisées de façon individuelle, avec ou sans interactions. Lorsque les stratégies concourent à des interactions, celles-ci peuvent se faire soit uniquement entre les étudiants, ce qui est souvent le cas lors de discussions en sous-groupes, soit être médiatisées par l'enseignant, dans le cas de discussions en plénière. Plusieurs auteurs ont proposé des stratégies ou des méthodes d'enseignement qui peuvent se retrouver dans ce groupe, elles sont amplement documentées dans la littérature (voir par exemple Sweat et Michealsen (2012), Barkley (2009), Angelo et Cross (1993), Schreiner et Louis (2011) ou encore Nilson (2010)).

Le deuxième groupe, surtout connu sous l'appellation inquiry-based learning (Minner, Levy et Century, 2009), que nous traduisons par " apprentissage inquisitif » ou encore par " apprentissage investigatif », recouvre des stratégies comme l'apprentissage par problème (Hmelo et Evenson, 2000), la méthode des cas (McNair et Hesum, 1954) et les projets de recherche de petite amplitude (Chu, 2009). L'étudiant est 
directement impliqué dans des activités de recherche, de manipulation, d'application et de résolution de problèmes ou de situations complexes. Ce groupe de pédagogies actives se situe vers le centre du continuum de Prince. La durée des activités d'apprentissage peut donc varier de quelques heures à quelques jours et impliquer du travail individuel ou en équipe. En plus de la durée des activités, ce qui distingue ce groupe du premier est en lien avec la nature des activités d'apprentissage proposées qui sont d'envergure plus grande et peuvent exiger la mobilisation de compétences en plus des habiletés cognitives (Prégent et al., 2009).

Le troisième groupe est connu en tant qu'experiential learning (Kolb, 1984), que l'on peut traduire par l'apprentissage expérientiel. Ce dernier groupe regroupe des pédagogies actives qui se situent du côté droit du continuum de Prince, car elles impliquent des activités et des tâches dont la réalisation demande considérablement de temps (des semaines, voire des mois). On peut penser à des stratégies d'enseignement comme l'apprentissage par projet (Barron, 1998), le jeu de rôle (Chamberland, et Provost, 1996), les simulations (Hertel et Millis, 2002) et les jeux sérieux (Alvarez et Djaouti, 2010). Ces pédagogies actives offrent aux étudiants la possibilité de se projeter ou d'assumer des rôles dans un contexte qui simule la pratique professionnelle. Cela leur permet de mettre en application les connaissances, les habiletés ou les compétences nécessaires pour résoudre des situations réelles du milieu où ils travailleront au terme de leur formation. Parce que l'ampleur ou la complexité des situations peuvent être très prononcées, l'enseignant peut décider de contrôler certaines variables afin de rendre possible la réalisation des tâches associées. Parmi les variables usuelles se trouvent la taille des équipes, la nature des livrables, le réalisme de la situation, le temps et l'aide impartie ou l'encadrement offert.

Rappelons que ces trois groupes ne sont ni complètement étanches ni mutuellement exclusifs. Ils suggèrent plutôt un moyen simple qui peut faciliter les décisions lors du choix des activités d'enseignement et d'apprentissage.Le tableau 1 présente les trois groupes d'apprentissage dans le cadre de pédagogies actives, donne une brève description de chacun et offre une liste non exhaustive d'exemples pour chaque groupe.

Tableau 1. Regroupement des pédagogies d'apprentissage actif

\begin{tabular}{|c|c|c|}
\hline $\begin{array}{c}\text { Apprentissage } \\
\text { réflexif-interactif }\end{array}$ & $\begin{array}{c}\text { Apprentissage } \\
\text { inquisitif }\end{array}$ & $\begin{array}{c}\text { Apprentissage } \\
\text { expérientiel }\end{array}$ \\
\hline $\begin{array}{c}\text { Activités de réflexion, de } \\
\text { discussion, d'analyse et } \\
\text { d'évaluation d'idées ou de } \\
\text { concepts. }\end{array}$ & $\begin{array}{c}\text { Activités de manipulation, } \\
\text { d'application et de } \\
\text { résolution de problèmes } \\
\text { complexes. }\end{array}$ & $\begin{array}{c}\text { Activités qui demandent aux } \\
\text { étudiants de jouer des rôles } \\
\text { qui simulent la pratique } \\
\text { professionnelle. }\end{array}$ \\
\hline Exemples de méthodes et de stratégies \\
\hline Team-based learning & $\begin{array}{c}\text { Apprentissage par } \\
\text { problème }\end{array}$ & Apprentissage par projet \\
\hline Think-pair-share & Méthode de cas & Simulations \\
\hline Discussions & Projet de recherche & Jeu de rôle \\
\hline Débats & Expérience en laboratoire & Jeux sérieux \\
\hline Questions conceptuelles & Puzzle & Stages \\
\hline Peer instruction & Enquête collaborative & Service learning \\
\hline
\end{tabular}




\section{Approche pédago-centrée des technologies}

Les technologies de l'information et de communication (TIC) renvoient, de façon générale, à toute technologie qui, lorsque combinée, permet aux personnes et aux organisations d'interagir dans et avec le monde numérique (digital world) (Ramey, 2012). Ces technologies incluent les logiciels (software) et l'appareillage (hardware), l'infrastructure qui permet d'accéder ou de stocker des données, ainsi que les outils de communication. Omniprésentes dans nos environnements sociaux et professionnels, ces technologie sont réussi, en quelques décennies, à modifier considérablement notre rapport aux activités quotidiennes, et le milieu universitaire ne fait pas exception (Limniou, Downes et Maskell, 2015). De ce fait, le fort potentiel éducatif des TICE fait que leur utilisation ne cesse de gagner en popularité, et ce, autant auprès des enseignants que des apprenants (Collin et Karsenti, 2013).Mais certains experts questionnent la nature éducative des TICE, notamment sous l'angle de la valeur qu'elles ajoutent aux gestes d'enseignement et au processus d'apprentissage. Collin (2013), par exemple, soulève la question des usages numériques éducatifs par rapport aux usages numériques en éducation, et prévient d'un possible amalgame qui ne rendrait pas justice à la valeur éducative que peuvent avoir les TICE, qu'elles soient utilisées en salle de classe ou à l'extérieur. De ce point de vue, il devient avantageux de conceptualiser les usages des TICE en ne les limitant pas à la seule situation éducative, mais de les considérer dans leur globalité dans une approche élargie qui met en rapport le contexte éducatif et le contexte socioculturel (Selwyn, 2010).

À ce sujet, Céci (2018) s'est penché sur l'utilisation des TICE en s'interrogeant sur leur potentiel à modifier la forme universitaire, c'est-à-dire les formes sociales qui traversent les institutions. II est d'avis que les technologies ont le potentiel de redéfinir l'acte pédagogique en amplifiant ses effets et en facilitant la réalisation des tâches. II précise toutefois que la simple substitution d'outils analogiques (craie, crayon, etc.) en faveur des TICE ne garantit pas à elle seule des résultats supérieurs. II est plutôt nécessaire de considérer la manière dont les TICE sont intégrées en contexte universitaire. II met ainsi en garde contre une approche uniquement techno-centrée, donnant en exemple l'installation par les administrateurs de tableaux blancs interactifs (TBI) dans de nombreuses salles de classe universitaires et qui demeurent sous-utilisées. À l'instar d'autres experts (Karsenti, 2016 ; Thibert, 2012), Céci (2018) positionne le débat de l'utilité des TICE, celle-ci étant parfois remise en question par certains acteurs du système éducatif, selon une perspective pédagogique. II mentionne que pour faire évoluer la forme universitaire à l'ère du numérique, le recours aux TICE devrait se faire en symbiose avec les dispositifs pédagogiques. II privilégie ainsi une approche pédago-centrée, où la compatibilité des TICE et des dispositifs pédagogiques vise l'amplification des gestes d'enseignement et des processus d'apprentissage. Cette amplification s'avère profitable lorsque s'opère une symbiose mutualiste et que la technologie est bien adaptée à la pédagogie, ce qu'il appelle la pédagogie techno-symbiotique.

Toutefois, partant de la prémisse que les TICE ne sont pas immanentes, les chercheurs constatent qu'un accompagnement techno-pédagogique s'avère nécessaire au développement professionnel des enseignants (Karsenti, 2016, Villeneuve et al., 2012). De surcroît, Céci (2018) suggère que cet accompagnement porte sur trois éléments fondamentaux, soit la formation aux pédagogies actives, les technologies et la culture numérique. Pour cet auteur, "les pédagogies les mieux adaptées aux technologies, donc aptes à devenir "symbiotiques », sont essentiellement les pédagogies actives, centrées sur l'apprenant et sa mise en activité cognitive sur les savoirs à acquérir (p.5).»ll rejoint ainsi les propos d'autres experts qui proposent d'étudier l'utilisation des TICE en tenant compte de considérations socioculturelles, comme des facteurs sociodémographiques, socioéconomiques et ethnoculturels, qui peuvent avoir des incidences sur l'utilisation des technologies en contexte scolaire (Bennett et Maton, 2010). 


\section{Utilisation des TICE en contexte universitaire}

La justification du choix de nous intéresser aux TICE utilisées en support aux pédagogies actives s'inscrit donc dans la foulée de l'approche pédago-centrée proposée par Céci (2018). En retour, cette approche exerce une influence sur le choix du modèle à retenir pour la classification de l'utilisation des TICE par les enseignants. À ce sujet, force est de constater qu'il existe de nombreux modèles ou typologies des applications ou des usages des TICE en éducation. À titre d'exemple, mentionnons les travaux de Basque et Lundgren-Cayrol (2002), de Collin et Karsenti (2012), ou encore ceux de LeverDuffy et McDonald (2011). La lecture de ces écrits a permis de dégager trois constats. Le premier, et probablement le plus significatif, est qu'ils mettent en lumière la difficulté de concevoir un système de classification universel des objets technologiques pour l'enseignement et l'apprentissage. Par exemple, certains modèles visent l'enseignement primaire ou secondaire (Romero et Laferrière, 2015), ou encore une TICE en particulier (notamment Fievez (2017) sur l'utilisation des tablettes en classe) ou un sousensemble de TICE (par exemple Dejardin, Bachy et Lebrun (2014) sur l'utilisation des outils de plateformes en ligne pour l'enseignement universitaire). Le second constat est que toute classification vient avec son assortiment d'avantages, mais aussi d'inconvénients qui limitent la transférabilité, car sont omis certains critères de sélection, par exemple. Le troisième constat, qui découle en quelque sorte des deux précédents, est que les classifications évoluent à mesure qu'évoluent les technologies, les contextes et les usages qu'on en fait. Le défi était donc de trouver un modèle suffisamment général et inclusif pour convenir à toute discipline universitaire. Dans ce contexte, nous avons considéré les TICE dans leur acception élargie pour qu'elles comprennent à la fois les types d'usage et les intentions pédagogiques de l'enseignant (Lebrun, 2011), ainsi que la nature de l'utilisation qu'on fait des outils numériques (Romero et al., 2016), par exemple l'utilisation de l'Internet, de divers autres outils et produits numériques, de courriels, de plateformes numériques (Moodle, par exemple) ou d'environnements d'apprentissage numériques, qui font partie des processus d'enseignement-apprentissage depuis plusieurs années (Bates, 2015). En outre, les applications Web, telles que les plateformes d'apprentissage en ligne, sont de plus en plus utilisées en pédagogie universitaire pour faciliter la communication et favoriser la collaboration entre les étudiants et les enseignants (Lameul, 2008).

Ainsi, le modèle de Lebrun (2011) permet de classer ces différents outils en fonction de trois types d'usages : transmissif, incitatif et interactif. Les usages transmissifs ont pour but que l'enseignant donne de l'information aux étudiants, par exemple les espaces d'affichage, les fichiers et les hyperliens vers des documents. On considère les usages incitatifs lorsque l'enseignant donne du travail aux étudiants, par exemple des exerciseurs, des séquences d'activités d'apprentissage en ligne ou l'envoi de documents des étudiants à l'enseignant. Les usages interactifs ont lieu lorsqu'il y a un dialogue autour d'une tâche, par exemple les forums de discussion, la conception de wiki, les outils de travail collaboratif.

Par ailleurs, le modèle proposé par Romero et son équipe (2016) pour l'utilisation des TICE en enseignement servira d'assise théorique lors de l'analyse des données. Ce modèle est particulièrement intéressant, car les différentes TICE sont déployées sur un continuum de pédagogies allant des plus passives aux plus actives (Romero, Laferrière et Power, 2016).Ce modèle s'avère ainsi très cohérent avec l'approche pédago-centrée, en plus de faire écho au continuum des pédagogies actives de Prince (2011). Le modèle propose cinq niveaux d'utilisation pédagogique des TIC. Les niveaux sont situés sur un continuum qui va d'une utilisation simple à une utilisation complexe et qui reflète les processus sociocognitifs dans lesquels les étudiants sont impliqués: 1-la consommation passive; 2- la consommation interactive; 3- la création de contenu; 4- la cocréation de contenu; 5- la cocréation participative de connaissances.

Les deux premiers niveaux sont de type consommation d'informations mises à la disposition des étudiants. Les apprenants peuvent soit lire ou visionner du contenu multimédia sans possibilité d'interaction avec ce contenu, soit interagir avec l'environnement numérique d'apprentissage, mais 
uniquement sur des options prédéfinies qui mènent vers des interactions basiques, généralement prédictibles et préprogrammées. Quelques exemples de ces niveaux sont les livres électroniques, les exercices en ligne, les tutoriels en ligne, tous disponibles via un clic sur une plateforme numérique. Le troisième niveau laisse la possibilité à l'étudiant de créer des textes, des images ou des vidéos liés à une situation d'apprentissage. La création de cartes conceptuelles, la programmation de jeux ludiques et l'écriture créative sont des exemples de ce niveau. Les deux derniers niveaux impliquent les étudiants dans un processus de co-création de contenu à partir de connaissances antérieures et de leur créativité pour trouver des solutions à des problèmes complexes, par l'entremise du travail collaboratif (utilisation d'un forum de discussion, collaboration pour créer un wiki) (Romero, Laferrière et Power, 2016). Le tableau suivant permet de jumeler les types d'usage principal des TICE proposés par Lebrun (2011) avec les niveaux d'utilisation des TICE du modèle proposé par Romero et son équipe (2016).

\section{Tableau 2. Types d'usage et utilisation des TICE en contexte universitaire}

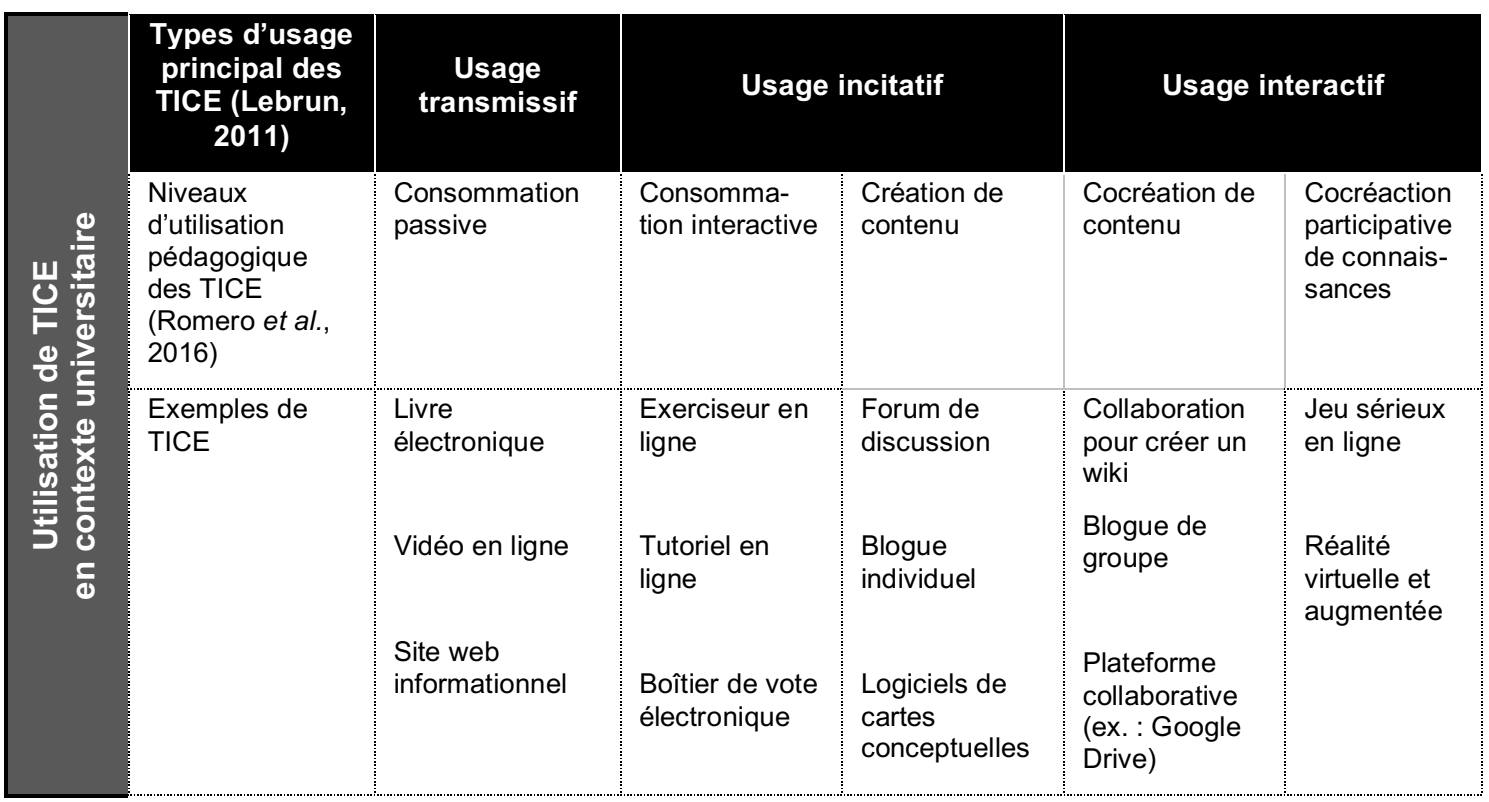

C'est dans la foulée de l'approche pédago-centrée que nous justifions le choix de nous intéresser aux TICE utilisées en support aux pédagogies actives, soit celles correspondant à l'usage dit interactif (engageant le dialogue autour d'une tâche) et non simplement à l'usage transmissif (visant à donner de l'information à l'étudiant) ou incitatif (visant à donner du travail à l'étudiant), selon la typologie des usages des outils des plateformes de soutien à l'enseignement universitaire proposée par Lebrun (2011). Rappelons que l'objectif principal de la présente étude est d'explorer quelles TICE sont utilisées lors des situations d'enseignement universitaire impliquant les pédagogies actives et surtout d'identifier à quel niveau d'utilisation elles appartiennent selon le modèle de Romero, Laferrière et Power (2016).

\section{Méthodologie}

Nous avons reçu l'aide des services pédagogiques de trois établissements universitaires de la région de Montréal(Canada) pour constituer notre échantillon. Nous nous sommes assurés que les trois services pédagogiques possèdent des listes d'envoi contenant l'adresse électronique d'enseignants (qu'ils soient professeurs ou chargés de cours) de leur université respective. Le statut professeur ou chargé de cours 
n'a pas été considéré pour cette recherche. Le seul critère de sélection était que le participant enseigne ou ait enseigné au moins un cours dans cet établissement. Les enseignants dont le nom paraissait sur ces listes ont reçu une invitation par courrier électronique pour répondre librement à un court questionnaire en ligne conçu pour cette enquête (voir les questions du questionnaire en appendice A).Ainsi, selon l'information reçue, ce sont environ 900 enseignants qui ont été sollicités. De ce nombre, nous avons reçu 153 questionnaires remplis ou partiellement remplis. Après avoir éliminé ces derniers, nous avons pu constituer un échantillon de 119 répondants, répartis dans six domaines d'études universitaires (gestion/administration, éducation/psychologie, droit, sciences humaines et sociales, sciences/technologies et génie, sciences de la santé). Même s'il contient des enseignants provenant de différentes universités et d'une variété de domaines, cet échantillon de convenance ne prétend pas être représentatif de l'ensemble des enseignants universitaires du Québec. II faudra tenir compte de cette importante limite méthodologique lors de la discussion des résultats.

Le questionnaire est centré sur le recours aux méthodes ou aux stratégies d'enseignement considérées comme étant des pédagogies actives ainsi que sur l'utilisation des TICE en support à ces stratégies d'enseignement. Le questionnaire comprend des questions à choix multiples sur l'utilisation de pédagogies actives et des TICE au service de celles-ci, et quelques questions à réponse courte. Des données permettant de tenir compte de certains facteurs complémentaires ont également été recueillies, notamment le genre, le nombre d'années d'expérience en enseignement, la discipline enseignée, le type de formation suivie en pédagogie ou en TIC, le niveau de compétence déclaré en TIC et le profil d'utilisateur de TICE selon le nombre d'outils de TICE utilisés dans un cours en moyenne (6 TICE ou moins étant considéré un petit utilisateur, entre 7 et 12 TICE étant un moyen utilisateur, 13 TICE et plus étant un grand utilisateur).

Les données recueillies ont d'abord été analysées avec des statistiques descriptives. Puis, dans une perspective exploratoire, nous avons réalisé des analyses en composantes principales (ACP) afin d'identifier les associations entre les variables à l'étude. L'ACP permet ainsi de visualiser la position des individus sur des axes en fonction de variables qui les rassemblent. Plusieurs avantages sont associés à cette méthode, notamment sa simplicité, sa flexibilité et sa puissance. De plus, elle fournit des graphiques permettant d'appréhender les résultats d'un simple coup d'œil. L'analyse des données descriptives et l'ACP ont été produites avec le logiciel SPSS. Seules les données des formulaires entièrement complétés ont été retenues.

\section{Résultats}

Puisque la posture de recherche adoptée pour l'analyse des données est exploratoire, nous présentons, en premier lieu, les données descriptives sous forme de tableaux de fréquence sur les deux construits principaux retenus, soit les pédagogies actives en contexte universitaire et l'utilisation de TICE en support à ces pédagogies actives. Ensuite, afin de dégager des tendances et de tracer les contours de profils potentiels d'enseignants universitaires quant à leurs pratiques déclarées en lien avec l'utilisation de TICE et de pédagogies actives dans le cadre de leur enseignement, nous présentons les résultats d'une analyse en composantes principales (ACP) et l'interprétation que nous en faisons.

\section{Utilisation de pédagogies actives en enseignement universitaire}

Concernant les pédagogies actives utilisées, tous les participants sauf un $(n=118)$ affirment les intégrer à leur enseignement universitaire. De plus, comme l'indique le tableau 3 ci-dessous, $28,6 \%$ des participants allouent peu de temps de cours aux pédagogies actives (de $11 \%$ à $25 \%$ du temps), 38,7 \% y allouent plus du quart de temps de cours (de $26 \%$ à $50 \%$ du temps) et $25,2 \%$ y allouent plus de la moitié du temps de cours (plus de $50 \%$ du temps). 
Tableau 3. Portion du cours universitaire allouée aux pédagogies actives (pratiques déclarées)

\begin{tabular}{ll}
\hline Portion du cours allouée aux pédagogies actives & $\boldsymbol{n = 1 1 8}$ \\
\hline $10 \%$ et moins du cours & $7,2 \%$ \\
De $11 \%$ à $25 \%$ du cours & $28,6 \%$ \\
De $26 \%$ à $50 \%$ du cours & $38,7 \%$ \\
Plus de $50 \%$ du cours & $25,2 \%$ \\
\hline
\end{tabular}

Concernant la variété des pédagogies actives utilisées selon les affirmations des participants ayant répondu au questionnaire, $85,7 \%$ disent utiliser entre 3 et 7 types de pédagogies actives parmi les choix proposés dans le questionnaire. Les types de pédagogies actives les plus souvent utilisées sont la méthode de cas $(79,8 \%)$, la discussion $(70,6 \%)$ et l'apprentissage par problème $(66,4 \%)$, comme le montre le tableau 4 ci-dessous, reprenant les types suggérés dans le questionnaire.

Tableau 4. Utilisation de chaque type de pédagogies actives (pratiques déclarées)

\begin{tabular}{lll}
\hline & Types ${ }^{1}$ de pédagogies actives utilisés & $\boldsymbol{n = 1 1 8}$ \\
\hline 1 & Méthode de cas & $79,8 \%$ \\
2 & Discussion & $70,6 \%$ \\
3 & Apprentissage par problème & $66,4 \%$ \\
4 & Apprentissage par projet & $53,8 \%$ \\
5 & Simulation & $40,3 \%$ \\
6 & Apprentissage collaboratif (team-based learning) & $37,8 \%$ \\
7 & Classe inversée (flipped classroom) & $36,1 \%$ \\
8 & Enseignement par les pairs (peer instruction) & $31,9 \%$ \\
9 & Apprentissage coopératif (puzzle) & $24,4 \%$ \\
10 & Jeu de rôle & $16,8 \%$ \\
11 & Par le jeu (game-based learning ou gamification) & $n=3$ \\
\hline
\end{tabular}

\section{Utilisation de TICE en support aux pédagogies actives en enseignement universitaire}

En réponse à la question «En classe, utilisez-vous les TICE lorsque vous avez recours à ces pédagogies actives ? », 79,8\% des participants ont répondu par l'affirmative $(n=92)$. Parmi les TICE utilisées en support aux pédagogies actives mentionnées par les participants, les plus fréquentes sont celles utilisées pour créer ou gérer des sondages (Socrative, Kahoot, Polleverywhere, GoogleForms, SurveyMonkey), comme l'indique le tableau 5 ci-dessous. Les enseignants mobilisent également des

${ }^{1}$ Il est à noter que des regroupements ont été faits pour intégrer des données « Autres » dans ces résultats. Notamment, la technique Fishbowl a été intégrée à la catégorie "Simulation », la technique Think-Pair-Share a été intégrée à la catégorie " Discussion », les techniques Challenge-Based Learning et Puzzle ont été intégrées à la catégorie « Apprentissage par problème », et la catégorie

" Apprentissage par le jeu » englobe Gamatronics, Gamification et Game-Based Learning. 
technologies qui permettent d'accéder à des ressources pédagogiques médiatisées par Internet (YouTube, AppleTV, Moodle). Puis, dans une moindre mesure, certains enseignants utilisent des réseaux sociaux et des outils technologiques permettant la réflexion et la cocréation de contenu (boîtier de vote, wiki).

Tableau 5. TICE utilisées en support aux pédagogies actives (pratiques déclarées des 92 répondants affirmant utiliser les TICE lorsqu'ils ont recours à des pédagogies actives dans leur enseignement universitaire)

\begin{tabular}{lll}
\hline \multicolumn{3}{l}{ TICE les plus utilisées } \\
\\
& & \\
\hline 1 & Sondage (Socrative, Kahoot, Poll Everywhere, & $n=43$ \\
& GoogleForms, SurveyMonkey) & \\
2 & Google (Classroom, Docs for Education, Notes, Search) & $n=32$ \\
3 & Vidéos (YouTube, cameras, Apple TV, iMovie, DVD) & $n=18$ \\
4 & Environnement numérique d'apprentissage (Moodle) & $n=17$ \\
5 & Réseaux sociaux (Facebook, Whatsapp, Instagram) & $n=11$ \\
6 & Plickers, Clickers & $n=11$ \\
7 & Babillard (Blackboard, Google Calendar) & $n=9$ \\
8 & Wiki & $n=3$ \\
\hline
\end{tabular}

Une autre question du questionnaire visait à établir le profil d'utilisation des TICE en support aux pédagogies actives des participants. Ceux-ci devaient indiquer s'ils se considéraient " petit utilisateur » (6 TICE ou moins), « moyen utilisateur » (de 7 à 12 TICE) ou " grand utilisateur » (13 TICE et plus). Les résultats sont présentés dans le tableau 6 ci-dessous.

Tableau 6. Utilisation déclarée des TICE en support aux pédagogies actives (pratiques déclarées des 92 répondants affirmant utiliser les TICE lorsqu'ils ont recours à des pédagogies actives dans leur enseignement universitaire)

\begin{tabular}{ll}
\hline Profil d'utilisateur des TICE déclaré & $\boldsymbol{n}=\mathbf{9 2}$ \\
\hline " petit utilisateur » (6 TICE ou moins) & $n=69$ \\
" moyen utilisateur » (de 7 à 12 TICE) & $n=26$ \\
" grand utilisateur » (13 TICE et plus) & $n=9$ \\
\hline
\end{tabular}

La lecture de ce tableau permet de constater que parmi les enseignants affirmant utiliser des TICE dans leurs cours universitaires, très peu se considèrent comme de grands utilisateurs de TICE. Ainsi, dans le cadre de l'analyse exploratoire présentée ci-dessous, les catégories «moyen utilisateur » et " grand utilisateur » ont été amalgamées.

\section{RÉSULTATS DE L’ANALYSE DE CORRESPONDANCES MULTIPLES (ACP)}

L'ACP a permis d'identifier deux principales dimensions relativement aux données recueillies. Le code de couleur associé aux acronymes, présents dans la légende de la figure 3 ci-dessous, permet de visualiser la constitution des deux dimensions. Ainsi, la dimension 1 discrimine les participants 
principalement sur les variables compétences en TIC, genre et utilisation des TICE. La dimension 2 discrimine selon les variables discipline, nombre d'années d'expérience en enseignement et type d'utilisateur des TICE. Partant de cette répartition, certains regroupements peuvent être avancés pour dégager des profils d'enseignants universitaires utilisant des TICE en support à des pédagogies actives dans leurs cours.

Figure 2. Résultats de l'analyse en composantes principales avec tracés de tendance proposés.

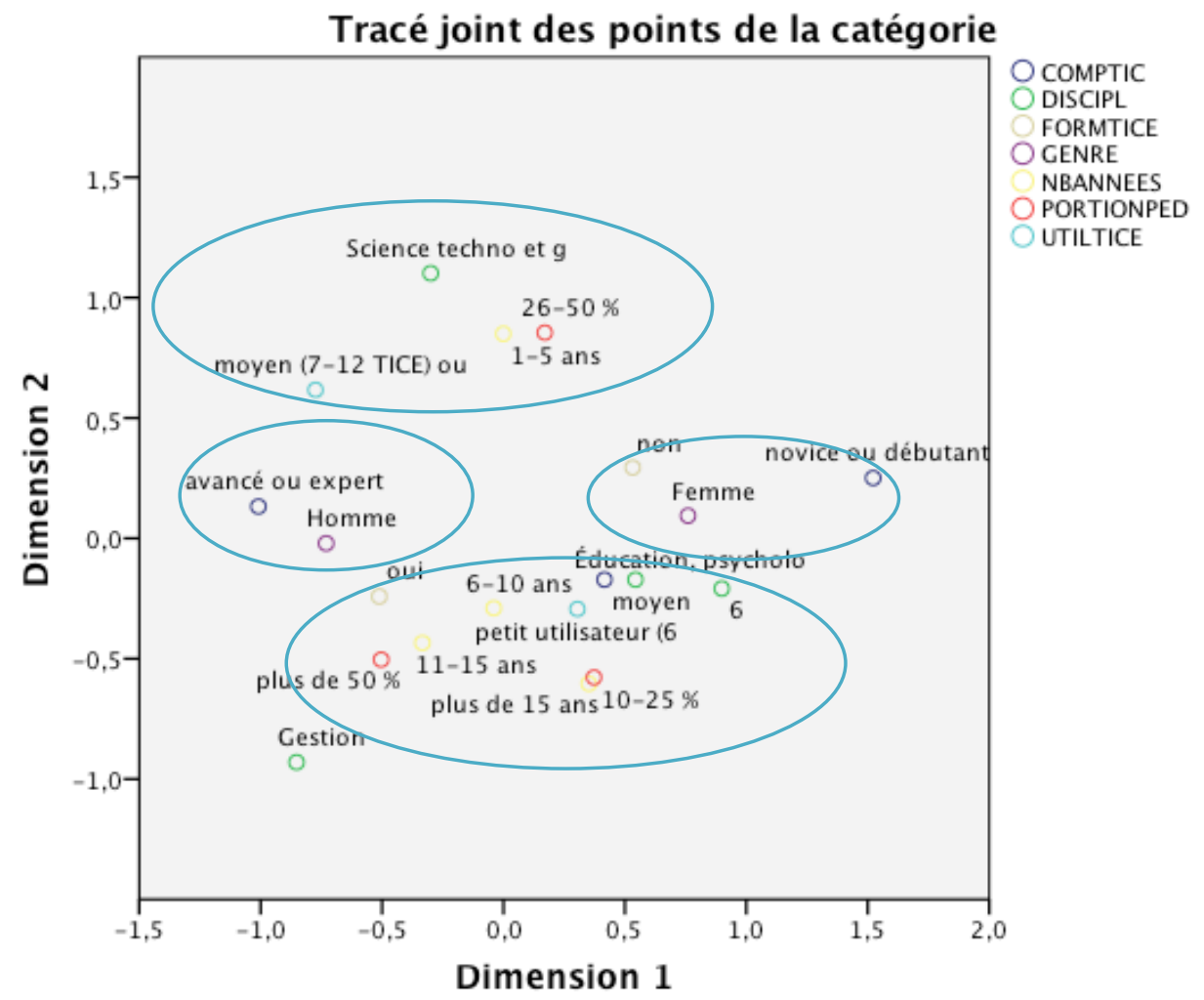

Normalisation variable principale.

Les profils que nous dégageons à partir de la dimension 2 sont les suivants, un étant plus restreint, l'autre plus général :

1) Un premier profil particulier semble se dessiner, soit celui d'enseignants ayant entre un et cinq ans d'expérience dans le domaine des sciences et technologies ou du génie, qui utiliseraient au moins sept TICE en support aux pédagogies actives, qu'ils proposent dans une proportion de 26 à $50 \%$ des cours universitaires qu'ils dispensent.

2) Un second profil, plus général, englobe les enseignants universitaires en éducation, en psychologie, en droit ou en sciences humaines et sociales, ayant au moins six ans d'expérience et considérant leur compétence en TIC comme étant moyenne; ceux-ci déclarent utiliser six TICE ou moins en support aux pédagogies actives qu'ils proposent dans leurs cours universitaires dans des proportions variables (moins de $25 \%$ du temps ou plus de $50 \%$ du temps). 
Comme on le remarque sur la figure 2, le genre ne semble pas être relié aux variables qui nous intéressent, soit la proportion du cours dédié aux pédagogies actives et le nombre de TICE utilisées.

Cependant, sur la dimension 1 de la figure2, pour ce qui est de l'utilisation de TICE, item où les participants devaient répondre par « oui » ou « non », nous dégageons deux profils où le genre est relié à la variable :

3) D'un côté, les hommes enseignant à l'université se disant avancés ou experts en TIC ont tendance à utiliser des TICE en soutien aux pédagogies actives dans leurs cours.

4) De l'autre côté, les femmes enseignant à l'université se disant novices ou débutantes en TIC ont tendance à ne pas utiliser de TICE en soutien aux pédagogies actives dans leurs cours.

\section{Discussion}

Les résultats présentés donnent un aperçu des pratiques pédagogiques universitaires telles que déclarées par des enseignants universitaires en ce qui a trait aux pédagogies actives et aux TICE en soutien à celles-ci. Une première observation qui émerge est qu'une forte majorité d'enseignants universitaires affirment avoir recours aux pédagogies actives. De surcroît, il est intéressant de constater qu'ils disent leur allouer une proportion non négligeable du temps de classe. Ces résultats vont dans le même sens que ceux d'études récentes (Kozanitis et Desbiens, 2016) qui suggèrent que les pratiques pédagogiques à l'université tendent à évoluer. La méthode traditionnelle de l'exposé magistral semble céder du terrain aux méthodes plus actives. De plus, en référence au regroupement présenté dans le tableau 1, les méthodes et stratégies d'enseignement les plus fréquemment utilisées appartiennent aux catégories apprentissage inquisitif et apprentissage expérientiel, toutes deux situées du côté pédo-centré du continuum de Prince (2004). La catégorie apprentissage interactif est elle aussi mise à contribution, notamment lors de discussions, ainsi que lors de l'utilisation de stratégies comme l'apprentissage collaboratif ou coopératif, ces derniers se situant vers le centre gauche du continuum. II est donc permis de penser que les méthodes d'enseignement magistro-centrées, comme l'exposé magistral, occupent toujours une place considérable dans les choix pédagogiques des enseignants, mais qu'elles semblent céder du terrain au profit de méthodes d'enseignement davantage pédo-centrées (Bachy, Lebrun et Smidts, 2010). Les données semblent même suggérer que les enseignants ont recours à un cocktail de méthodes d'enseignement lors d'un même cours, offrant de la sorte une diversité d'activités d'apprentissage. II serait intéressant, pour une prochaine recherche, de tenter d'identifier ce qui détermine le choix des méthodes et stratégies d'enseignement proposées.

Pour ce qui est de l'utilisation des technologies en lien avec les pédagogies actives, une forte proportion des enseignants universitaire ayant participé à l'enquête affirment les utiliser, même si la majorité d'entre eux se considèrent en être de petits utilisateurs, c'est-à-dire qu'ils emploient moins de six TICE pour leurs cours. Quoique nous ayons pu identifier les TICE utilisées par les enseignants, il aurait été intéressant de pouvoir vérifier quelle TICE est utilisée en fonction de quelle méthode d'apprentissage actif. Cela dit, les technologies qui permettent de sonder les étudiants en classe sont les plus fréquemment utilisées, ce qui est compatible avec des stratégies d'apprentissage actif parmi les plus populaires auprès de notre échantillon (apprentissage collaboratif et enseignement par les pairs). Cela signifie que la plupart des utilisations de TICE déclarées par les enseignants se retrouvent aux premier et deuxième niveaux selon la catégorisation de Romero, Laferrière et Power (2016). Certains enseignants semblent toutefois en faire un usage qui se situe à des niveaux supérieurs, notamment par l'utilisation des réseaux sociaux et du wiki. 
En examinant l'effet des autres variables sur l'utilisation des TICE, les résultats de l'ACP semblent indiquer que le genre pourrait être une variable sociodémographique liée à la fréquence d'utilisation, puisque les hommes apparaissent les utiliser davantage que les femmes. Par contre, on constate que le fait d'avoir suivi une formation en pédagogie ou en TICE est également lié à l'utilisation des TICE. Nous avons donc vérifié les données pour constater qu'une proportion plus importante d'hommes déclare avoir suivi ce genre de formation, ce qui pourrait expliquer en partie ces résultats. Ces résultats seraient également compatibles avec les études qui soulèvent l'importance la formation des enseignants pour l'utilisation des TICE (Karsenti, 2016, Villeneuve et al., 2012).II sera nécessaire d'approfondir les recherches à ce sujet en réalisant des entrevues auprès des enseignants pour confirmer ces hypothèses.

Par ailleurs, dans une étude de Dejardin, Bachy et Lebrun (2014) sur l'utilisation de plateformes d'apprentissage en ligne (correspondant à l'utilisation de la plateforme Moodle, notamment, en contexte universitaire québécois) chez des enseignants du supérieur belges, la variable discipline d'enseignement (classifiée selon la typologie de Biglan-Becher (1989), citée dans Berthiaume (2007)) était corrélée à certains résultats, notamment l'utilisation de l'outil Travaux des plateformes d'apprentissage, soit le seul outil que les auteurs associent à une forme d'interactivité dans l'enseignement supérieur selon la typologie de Lebrun (2011), qui était en lien avec les disciplines Hard-Applied (correspondant au génie civil) et Soft-Applied (correspondant au travail social), soit des sciences appliquées. Dans la présente étude, quoique nous n'ayons pas pu utiliser la même typologie des disciplines d'enseignement, certaines tendances se sont dessinées en lien avec ce facteur. En effet, les personnes enseignant en sciences et technologies ou en génie, disciplines qui se retrouveraient dans le pôle Hard-Applied, semblent utiliser davantage les TICE en support aux pédagogies actives que leurs pairs des autres disciplines. Ce résultat est conforme à celui rapporté par Guennoun et Benjelloun (2016), qui constataient des différences selon les disciplines.

\section{LIMITES DE L’ÉTUDE}

Certains aspects de l'outil de collecte de données, soit le questionnaire, représentent des limites sur le plan de la validité, notamment pour ce qui est des conceptions des enseignants universitaires et de leur adéquation aux types de pédagogies actives présentes comme choix dans le questionnaire et à l'aspect "support aux pédagogies actives" des questions sur l'utilisation des TICE. En effet, comme nous l'avons exposé plus haut, de nombreuses typologies de pédagogies actives existent. Par exemple, la compréhension d'un participant sur ce que constitue une discussion, dans un paradigme actif, peut différer grandement de la compréhension d'un autre participant ou de celle des chercheurs.

D'autre part, les questions du questionnaire portant sur l'utilisation des TICE concernaient seulement les TICE effectivement utilisées en soutien aux pédagogies actives. Or, selon les exemples donnés par les participants qui disent utiliser des TICE, cet aspect important de la question semble ne pas avoir été considéré dans leurs réponses; en effet, le courriel et les moteurs de recherche sur Internet semblent peu propices à soutenir les pédagogies actives. II est donc pertinent de se demander dans quelles mesures les TICE mentionnées soutiennent effectivement des pédagogies actives et non pas simplement la logistique d'un cours universitaire. D'ailleurs, la majorité des outils des plateformes d'apprentissage utilisés en cours universitaire en Belgique, selon l'étude de Dejardin, Bachy et Lebrun (2014), ne visent pas l'interaction (un seul des outils recensés, soit Travaux), mais visent plutôt des usages transmissifs, où l'enseignant donne de l'information à l'étudiant, et incitatifs, où l'enseignant donne du travail à l'étudiant, ces deux types d'usages n'étant pas considérés comme des pédagogies actives selon notre cadre conceptuel.

De plus, les pratiques déclarées des enseignants sont loin de refléter les pratiques réelles en salle de classe. Ainsi, dans de futures recherches, il serait intéressant d'intégrer à la fois les conceptions des enseignants par rapport aux pédagogies actives et aux TICE en soutien à celles-ci, et des artéfacts démontrant des pratiques de pédagogies actives avec TICE utilisées en enseignement supérieur. 


\section{Conclusion}

En conclusion, bien que les enseignants d'université utilisent régulièrement les TICE, la plupart d'entre eux se limitent à une simple utilisation des TICE, c'est-à-dire à la transmission de l'information (Kirkup et Kirkwood, 2005) ou, du côté des étudiants, à la consommation passive (Romero et Laferrière, 2015). Dans le contexte de l'augmentation de l'utilisation à la fois des TICE et des pédagogies actives en contexte universitaire, il semble opportun, dans le cadre de recherches futures, de se demander dans quelle mesure les TICE enrichissent, améliorent ou approfondissent l'apprentissage qui a lieu à l'université. Du point de vue des étudiants, ce qu'il faut vraiment considérer, ce n'est pas de savoir si les enseignants utilisent les TICE, mais plutôt comment ils les utilisent. La présente étude exploratoire a tenté d'apporter un premier aperçu en réponse à ces dernières questions. II convient maintenant de creuser davantage les facteurs et les modalités qui rendent efficiente l'utilisation de TICE en support aux pédagogies actives en contexte universitaire.

De plus, il semble pertinent de continuer à fournir une offre de formation en TICE pour les enseignants universitaires afin de les soutenir dans leurs efforts d'intégrer les TICE en soutien aux pédagogies actives qu'ils utilisent déjà dans leurs cours.

\section{Bibliographie}

ALVAREZ, J. et DJAOUTI, D. (2010). Introduction au Serious game. Paris: Questions théoriques.

ANGELO, T. A. et CROSS, K. P. (1993). Classroom Assessment Techniques: A Handbook for College Teachers (2e ed.). San Francisco: Jossey-Bass.

BACHY S., LEBRUN, M. et SMIDTS, D. (2010). Un modèle-outil pour fonder l'évaluation en pédagogie active : impact d'une formation sur le développement professionnel des enseignants ». Revue internationale de pédagogie de l'enseignement supérieur [En ligne] : http://ripes.revues.org/307.

BARKLEY, E. (2010). Student engagement techniques: A handbook for college faculty. San Francisco: Jossey-Bass.

BARRON, B. (1998). Doing with understanding: Lessons from research on problem and project-based learning. Journal of Learning Science, vol. 7, no 3-4, p. 271-311.

BASQUE, J. et LUNDGREN-CAYROL, K. (2002). Une typologie des typologies des applications des TIC en éducation. Sciences et techniques éducatives, vol. 9, no 3-4, p. 263-289.

BATTALIO, J. (2010). Success in distance education: Do learning styles and multiple formats matter? American Journal of Distance Education, vol. 23, no 2, p. 71-87.

BATES, A.T. (2015). Teaching in a Digital Age. Guidelines for designing teaching and learning. En ligne : https://teachonline.ca/sites/default/files/pdfs/teaching-in-a-digital-age_2016.pdf.

BENNETT, S. et MATON, K. (2010). Beyond the "digital natives" debate: Towards a more nuanced understanding of students' technology experiences. Journal of Computer Assisted Learning, vol. 26, no 5, p. 321-331.

BONWELL, C.C. et EISEN, J.A. (1991). Active learning: Creating excitement in the classroom. ASHE-ERIC Higher Education Reports, no 1. Washington, DC : George Washington University.

BROOKS, C.D. (2016). ECAR Study of Undergraduate Students and Information Technology. Research report. Louisville, CO.

BUCKLEY, C. A., PITT, E., NORTON, B., et OWENS, T. (2010). Students' approaches to study, conceptions of learning and judgements about the value of networked technologies. Active Learning in Higher Education, vol. 11, no. 1, p. 55-65.

CARR, R., PALMER, S. et HAGEL, P. (2015). Active learning: the importance of developing a comprehensive measure. Active learning in higher education, vol. 16, no 3, p. 173-186.

CÉCI, J-F. (2018). Les technologies peuvent-elles modifier la forme universitaire ? Certainement ! Distances et médiations des savoirs. En ligne : http://journals.openedition.org/dms/2356.

revue-mediations.teluq.ca | vol. 01, no. 1 
CHAMBERLAND, G. et PROVOST, G. (1996). Jeu, simulation et jeu de rôle. Sainte-Foy: Les Presses de l'Université du Québec.

CHARBONNEAU, L. (2013). More on student preferences: good lectures vs. classroom technology. University Affaires. En ligne : https://www.universityaffairs.ca/news/news-article/more-on-student-preferences-good-lectures-vsclassroom-technologyl.

COLLIN, S. et KARSENTI, T. (2013). Usage des technologies en éducation: analyse des enjeux socioculturels. Éducation et francophonie, vol. 41, no 1, p. 192-210. En ligne : http://www.acelf.ca/c/revue/pdf/EF-41-1-192_COLLIN.p.

COLLIN, S., GUICHON, N. et NTEBUTSE, J. G. (2015). Une approche sociocritique des usages numériques en éducation. STICEF, vol. 22, p.1-29.

DEJARDIN, S., BACHY, S. et LEBRUN, M. (2014). Le profil de l'enseignant de l'enseignement supérieur influence-t-il l'usage des outils des plateformes? International Journal of Technologies in Higher Education, vol. 11, no 2, p. 19-34.

DUFFY, J. et McDONALD, J. (2011). Teaching and learning with technology. Boston: Pearson Education, Inc.

FIÉVEZ, A. (2017). L'intégration des TIC en contexte éducatif : modèles, réalites et enjeux. Québec : Presses de l'Université du Québec.

FREEMAN, S., EDDY, S. L., MCDONOUGH, M., SMITH, M. K., OKOROAFOR, N., JORDT, H. et WENDEROTH, M. P. (2014). Active learning increases student performance in science, engineering, and mathematics. PNAS, vol. 111, no 23, p. 8410-8415.

GUENNOUN, B. et BENJELLOUN, N. (2016). Regards des étudiants sur l'intégration des TIC dans l'enseignement supérieur scientifique. Revue internationale des technologies en pédagogie universitaire, vol. 13, no 1, p. 64-94.

HERTEL, J.P. et MILLIS, B.J. (2002). Using simulations to promote learning in higher education: an introduction. Sterling, VA: Stylus Publishers.

HIGGINS, S., XIAO, Z.et KATSIPATAKI, M. (2012). The impact of digital technology on learning: A summary for the education endowment foundation. En ligne : https://educationendowmentfoundation.org.uk/public/files/Publications/The_Impact_of_Digital_Technologies_on_ Learning_(2012).pdf.

HMELO, C.E. et EVENSEN, D.H. (2000). Introduction Problem-based Learning: Gaining Insights on Learning Interactions through Multiple Methods of Inquiry. In D.H. Evensen and C.E. Hmelo (eds), Problem-based Learning a Research Perspective on Learning Interactions (). Mahwah, New Jersey: Lawrence Erlbaum Associates, p. 227-248.

INTERNATIONAL ASSOCIATION FOR THE EVALUATION OF EDUCATIONAL ACHIEVEMENT (IEA). (2013). International computer and information literacy study: Assessment framework. En ligne, http://www.iea.nl/fileadmin/user_upload/Publications/Electronic_versions/ICILS_2013_Framework.pdf.

JOHNSON, D, W. et JOHNSON, R.T. (2014). Using technology to revolutionize cooperative learning: an opinion. Frontiers in Psychology. En ligne, DOI: 10.3389/fpsyg.2014.01156.

KARSENTI, T. (2016). Les technologies ont-elles un réel impact sur la réussite scolaire ? En ligne : http://www.cforp.ca/educo/les-technologies-ont-elles-un-reel-impact-sur-la-reussite-scolaire/\#_ftn7.

KARSENTI, T. et COLLIN, S. (2011). L'enseignement-apprentissage 2.0 : la nécessité d'apprivoiser les technologies émergentes en enseignement supérieur! Revue de la Haute École Pédagogique de Berne, du Jura et de Neuchâtel, vol. 16. p.16-18.

KIRKUP, G. et KIRKWOOD, A.(2007). Information and communications technologies (ICT) in higher education teaching-a tale of gradualism rather than revolution. Learning, Media and Technology, vol. 30, no 2, p.185-199.

KNIGHT, J. (2010). Distinguishing the learning approaches adopted by undergraduates in their use of online resources. Active Learning in Higher Education, vol.11, no 1, p. 67-76.

KOLB, D. A. (1984). Experiential learning: Experience as the source of learning and development. Englewood Cliffs, N.J.: Prentice-Hall Inc.

KOZANITIS, A. et DESBIENS, J.-F. (2016). Exploring the combined effects of internal and external sources of motivation in the context of an outcome-based education for Canadian engineering Students. International Journal of Engineering Education, vol. 32, no 5(A), p. 1847-1858.

LAMEUL, G. (2008). Les effets de l'usage des technologies d'information et de communication en formation d'enseignants, sur la construction des postures professionnelles. Savoirs, no 17, p. 71-94. 
LEBRUN, M. (2011). Impacts des TIC sur la qualité des apprentissages des étudiants et le développement professionnel des enseignants : vers une approche systémique. Sciences et technologies de l'information et de la communication pour l'éducation et la formation (Sticef), no 18. En ligne, http://sticef.univ-lemans.fr.

LIMNIOU, M., DOWNES, J. et MASKELL, S. (2015). Datasets reflecting students' and teachers' views on the use of learning technology in a UK university. British Journal of Educational Technology, vol. 46, no. 5, p.1081-1091.

LEVER-DUFFY, J. et MCDONALD, J. (2011). Teaching and learning with technology. Boston: Pearson Education.

MINNER, D., LEVY, A.J. et CENTURY, J. (2009). Inquiry-based science instruction- what is it and does it matter? Results from a research synthesis years 1984-2002. Journal of Research in Science Teaching, vol. 47, no 4, p. 474-496.

MONSAKUL, J. (2008). A research synthesis of instructional technology in higher education, in K. McFerrin et al. (dir.), Proceedings of Society for Information Technology \& Teacher Education (SITE) International Conference 2008. Chesapeake, VA: Association for the Advancement of Computing in Education (AACE), p. 2134-2139.

NILSON, L.B. (2010). Teaching at its best: A research-based resource for college instructors (3e éd.). Bolton, MA: Anker Publishing Company, Inc.

PAIVANDI, S. et ESPINOSA, G. (2013). Les TIC et la relation entre enseignants et étudiants à l'université. Distances et médiations des savoirs. En ligne : $\underline{\text { http://journals.openedition.org/dms/425. }}$

PETRESS, K. (2008). What Is Meant By “Active Learning”? Education, vol. 128, no 4, p. 566-570.

PRÉGENT, R., BERNARD, H. et KOZANITIS, A. (2009). Enseigner à l'université dans une approche-programme. Un défi à relever. Montréal: Presses Polytechnique Montréal.

PRINCE, M. (2004). Does Active Learning Work? A Review of the Research. Journal of Engineering Education, July 2004, p. 223-231.

RABY, C., KARSENTI, T., MEUNIER, H. et VILLENEUVE, S. (2011). Usage des TIC en pédagogie universitaire : point de vue des étudiants. Revue internationale des technologies en pédagogie universitaire, vol. 3, p. 6-19.

RAMEY, K. (2012). Types of technology used in the classroom. Use of Technology. En ligne : https://www.useoftechnology.com/types-technology-classroom/.

ROGERS, E. M. (2001). The digital divide. Convergence: The international journal of Research into new media technologies, vol. 7 , no 4, p. 96-111.

ROGERS, G. (2004). History, learning technology and student achievement: Making the difference? Active Learning in Higher Education, vol. 5, no 3, p. 232-247.

ROMERO, M. (2017). Les compétences pour XXIe siècle, in M. Romero, B. Lille et A. Patiño (dir.), Usages créatifs du numérique pour l'apprentissage au XXl' siècle, Québec : Presses de l'Université du Québec, p. 15-28.

ROMERO, M. et LAFERRIĖRE, T. (2015). Usage pédagogique des TIC : de la consommation passive à la cocréation participative. VTÉ - Vitrine Technologie Éducation, p. 1-7.

ROMERO, M., LAFERRIĖRE, T. et POWER, T.M. (2016). The move is on! From the passive multimedia learner to the engaged co-creator. eLearn, vol. 3, no 1.En ligne: https://elearnmag.acm.org/archive.cfm?aid=2893358.

ROMERO, M., LILLE, B. et PATIÑO, A. (2017). Usages créatifs du numérique pour l'apprentissage au XXle siècle. Presses de l'Université du Québec.

ROUSSEL, C., LEMIEUX, M.-M., LANDRY, N., et SAMSON, G. (2017). L'utilisation du manuel numérique en contexte postsecondaire : avantages et inconvénients. Sticef, vol. 24, no 3. En ligne : doi:10.23709/sticef.24.3.1.

SELWYN, N. (2010). Looking beyond learning: Notes towards the critical study of educational technology. Journal of Computer Assisted Learning, vol. 26, no 1, p. 65-73.

SIMARD, S. KARSENTI, T. et MOTTET, M. (2017). Les compétences informationnelles : des pistes pédagogiques, in T. Karsenti et J. Bugmann (dir.), Enseigner et apprendre avec le numérique, Montréal : Presses de l'Université de Montréal, p. 73-86.

SCHREINER, L.A. et LOUIS' M. (2011). The engaged learning index. Implications for faculty development. Journal on Excellence in College Teaching, vol. 22, no 1, p. 5-28.

SMART, K. L., et CSAPO, N. (2007). Learning by doing: engaging students through learner-centered activities. Business Communication Quarterly, décembre, p. 451-457.

SWEET, M. S. et MICHAELSEN, L. K. (2012). Team-Based Learning in the Social Sciences and Humanities: Group Work that Works to Generate Critical Thinking and Engagement. Sterling, VA: Stylus. 
TESSIER, R. (2012). Pour une intégration pédagogique des TIC en classe. BDTP.

TURNEY, C.S., ROBINSON, D., LEE, M. et SOUTAR, A. (2009). Using technology to direct learning in higher education. The way forward? Active Learning in Higher Education, vol. 10, no 1, p. 71-83.

VILLENEUVE, S., KARSENTI, T., RABY, C. et MEUNIER, H. (2012). Les futurs enseignants du Québec sont-ils technocompétents? Une analyse de la compétence professionnelle à intégrer les TIC. Revue internationale des technologies en pédagogie universitaire, vol. 9, no 1-2, p. 78-99.

VENKATESH, V., RABAH, J., FUSARO, M., COUTURE, A., VARELA, W. et ALEXANDER, K. (2015). Factors impacting University instructors' and students' perceptions of course effectiveness and technology integration in the age of Web 2.0. McGill Journal of Education, vol. 5, no 1. En ligne: http://mje.mcgill.ca/article/view/9130/7093.

WANNER, T. (2015). Enhancing student engagement and active learning through just-in-time teaching and the use of PowerPoint. International Journal of Teaching and Learning in Higher Education, vol 27, no 1, p. 154-163. 


\section{Appendice A}

Questions du questionnaire en ligne envoyé aux participants :

\section{ENQUÊTE EXPLORATOIRE SUR L'UTILISATION DES TICEET DES PÉDAGOGIES ACTIVES}

Le but de ce court questionnaire est d'explorer les raisons pouvant expliquerla volonté des enseignants et des enseignantes d'utiliser les technologies de l'information et de communication en enseignement (TICE) en classe, lorsqu'ils ou elles ont recours aux pédagogies activesdans un contexte d'enseignement en présentiel ou hybride.

1) En classe, avez-vous recours à au moins une méthode ou stratégie d'enseignement considérée comme étant une pédagogie active?

Oui $\square \quad$ allez à la question 2

Non $\square \quad$ allez à la question 4

2) Si oui, laquelle ou lesquelles? (indiquez à l'aide d'un $x$ )

\begin{tabular}{|l|l|l|l|}
\hline Apprentissage par problème & & Apprentissage par projet & \\
\hline Méthodes des cas & & Apprentissage coopératif \\
\hline Simulation & & Discussion \\
\hline $\begin{array}{l}\text { Classe inversée } \\
\text { (flippedclassroom) }\end{array}$ & $\begin{array}{l}\text { Enseignement par les pairs (Peer } \\
\text { instruction) }\end{array}$ & \\
\hline Team-basedlearning & & Jeu de rôle & \\
\hline
\end{tabular}

Autre(s) (précisez) :

3) Quelle portion approximative du cours est allouée aux pédagogies actives?
$0-10 \% \square$
$11-25 \% \square$
$26-50 \%$
plus de $50 \%$

4) En classe, utilisez-vous les TICE lorsque vous avez recours àcette (ces) pédagogie(s) active(s)?

Oui, parfois ou très souvent $\square$ allez à la question 6(verso)

Non, jamais ou presque jamais $\square$ allez à la question 7 (verso)

5)Laquelle ou lesquelles utilisezvous? 
6) Décrivez brièvement quelle utilisation vous en faites.

7) Si vous avez répondu non à la question 4, pour quelle(s) raison(s)?

\begin{tabular}{|l|l|l|l|}
\hline Vous n'en connaissez pas & & Par manque de temps \\
\hline $\begin{array}{l}\text { Trop grande quantité de } \\
\text { contenu }\end{array}$ & & $\begin{array}{l}\text { Peu ou aucune valeur ajoutée } \\
\text { anticipée }\end{array}$ & \\
\hline $\begin{array}{l}\text { Ne savez pas comment les } \\
\text { TICE peuvent s'appliquer dans } \\
\text { un cours }\end{array}$ & & $\begin{array}{l}\text { Vous doutez de leur efficacité pour } \\
\text { améliorer l'apprentissage }\end{array}$ & \\
\hline $\begin{array}{l}\text { Les TICE ne s'appliquent pas à } \\
\text { votre (vos) matière(s) ou } \\
\text { cours }\end{array}$ & & $\begin{array}{l}\text { Vous craignezle risque qu'elles vous } \\
\text { lâchent durant le cours }\end{array}$ & $\begin{array}{l}\text { Les étudiants n'ont pas tous accès } \\
\text { aux outils technologiques } \\
\text { nécessaires }\end{array}$ \\
\hline $\begin{array}{l}\text { Les TICE risquent de nuire au } \\
\text { bon fonctionnement du cours }\end{array}$ & & \\
\hline
\end{tabular}

Autre(s) raison(s) (précisez) :

\section{INFORMATIONS SOCIODÉMOGRAPHIQUES}

8) Nombre d'années d'expérience en enseignement:

9) Discipline ou matière enseignée :

10) Avez-vous suivi une formation en pédagogie?

11) Si oui, durée ou nature :

12) Avez-vous suivi une formation pour l'utilisation des TICE pour l'enseignement?

13) Vous êtes : un homme $\square$ une femme préférez ne pas répondre

14) Votre de niveau de compétence en informatique :
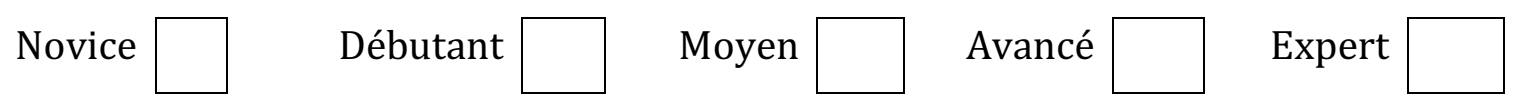

15) Indiquez votre profil d'utilisateur TICE, en fonction du nombre de technologies utilisées dans un cours en moyenne (logiciel de traitement de texte, logiciel de présentation, courriel, moteur de recherche sur Internet (Google), plateforme 
d'enseignement (Moodle), sites web liés au contenu de votre cours, DVD, planification de rencontre (Doodle), Forum de discussion, logiciel d'enregistrement audio-vidéo et de capture d'écran (Camtasia, Adobe Connect), outil de recherche bibliographique (Repère, Copernic), réseaux sociaux (Twitter, Facebook), Wiki, etc.)

Petit utilisateur $(6$ tech. ou moins) Moyen utilisateur (entre 7 et 12 tech.) Grand utilisateur (13 tech. ou plus)

Nous vous remercions chaleureusement pour votre collaboration. 